\title{
Structural Transition and Thermal Anomaly in $\mathrm{LiTlSO}_{4}$
}

\author{
Hiroyuki MashiYama, Jiyou Wu, \\ Fuminao Shimizu ${ }^{1}$ and Masaaki TAKASHige ${ }^{1}$ \\ Department of Physics, Faculty of Science, Yamaguchi University, 1677-1 Yoshida, Yamaguchi 753, Japan \\ ${ }^{1}$ College of Science and Engineering, Iwaki Meisei University, 5-5-1 Iino, Chuohdai, Iwaki 970, Japan
}

(Received September 4, 1997 )

KEYWORDS: $\mathrm{LiTISO}_{4}$, phase transition, thermal anomaly

A survey for discovering new ferroelectric or ferroelastic crystals has been worked in order to get more useful or functional materials or to understand the mechanism of ferroelectricity and ferroelasticity. In the last two decades, a family of crystals with a chemical formula $A_{2} B X_{4}$ have been widely investigated, because they have incommensurate phases. ${ }^{1)}$

If two alkali metal ions $A$ 's are not the same kind, then the crystal structure and the transition sequence become a little different from the $A_{2} B X_{4}$ family. ${ }^{1)}$ Among such $A A^{\prime} B X_{4}$-type crystals, $\mathrm{LiNH}_{4} \mathrm{SO}_{4}{ }^{2}$ ) and $\mathrm{LiRbSO}_{4}{ }^{3)}$ are ferroelectrics and take a general tridymite structure. ${ }^{4,5)}$ At high temperature, both crystals belong to an orthorhombic Pmcn $(Z=4)$ system, where each $\mathrm{SO}_{4}$ tetrahedron occupies two configurations with equal probability. ${ }^{6,7)}$ Ordering of the disordered tetrahedra takes place with lowering temperature. In $\mathrm{LiRbSO}_{4}$, incommensurately and commensurately modulated phases are realized above room temperature. ${ }^{8)}$ The $\mathrm{LiNH}_{4} \mathrm{SO}_{4}$ crystal is ferroelectric in the room-temperature phase and takes a superstructure below room temperature. ${ }^{9)}$

Recently Shimizu and Takashige synthesized $\mathrm{LiTlSO}_{4}$ by a Bridgman method. ${ }^{10)}$ They performed differential thermal analyses and observed two anomalies on cooling and one anomaly on heating, which indicates structural transitions of the crystal. They also measured dielectric constants; the constant perpendicular to the cleavage plane showed three and two anomalies (stepwise change or hump) on cooling and heating runs, respectively. The crystal was considered not to be ferroelectric because no $D E$-hysteresis loop was observable.

According to a preliminary study by X-ray scattering, ${ }^{11)}$ the room-temperature phase of the crystal belongs to Pmcn. In the low temperature region, superstructure reflections have been found. The diffraction pattern in the $(h k 0)$ zone seems to be a hexagonal one in the lowest temperature phase, although the degree of crystal symmetry usually decreases with decreasing

\footnotetext{
* On leave of absence from: Department of Photoelectrics and Information Engineering, Shandong University, Jinan, Shandong 250100, China
} 
temperature. In $A_{2} B X_{4}$-type crystals, flopping of $B X_{4}$ tetrahedra is necessary to become hexagonal from orthorhombic. ${ }^{12)}$ If such flopping takes place at the transition of $\mathrm{LiTlSO}_{4}$, then a large thermal anomaly will be observable. The character of the intermediate phase may also be clarified by thermal analyses. Since no further study has been reported so far as the author's knowledge, we investigated the transition entropy of this crystal.

Single crystals of $\mathrm{LiTlSO}_{4}$ were grown by the Bridgman method. The crystal is transparent and colorless. A small amount of crystal was sealed in an aluminum cell and was provided for the thermal analysis. Two kind of samples were prepared; powder of the crystal: $17.87 \mathrm{mg}$, and a peace of block cut from the bulk: $2.53 \mathrm{mg}$. Since the peace was selected from a single crystal part, it is called as a single crystal hereafter. A differential scanning calorimeter (Rigaku: DSC8230) was used. The heating rate was $10 \mathrm{~K} / \mathrm{min}$. The transition temperature was determined by a cross point of the slope line of the anomalous heat flow and the back ground line. The transition entropy was estimated from the amount of the excess heat flow.

With decreasing temperature, a differential thermal analysis was recorded on a chart. For the powder sample, two signals were detected; a small anomaly was at $267 \mathrm{~K}$ and a large anomaly peaked at $232 \mathrm{~K}$ with accompanying a shoulder around $238 \mathrm{~K}$ in similar to the first report in ref. 10 . For the single crystal, two anomalies were observed; a small one at $267 \mathrm{~K}$ and a large one at $243 \mathrm{~K}$. The second transition temperature depended on a cooling rate a little; it was about $253 \mathrm{~K}$ under a very slow cooling.

On heating, the excess heat flow at the structural transition was recorded as DSC signal, which is shown in Fig. 1 for the powder sample. We can notice two small anomalies at $236 \mathrm{~K}$ and $263 \mathrm{~K}$ and

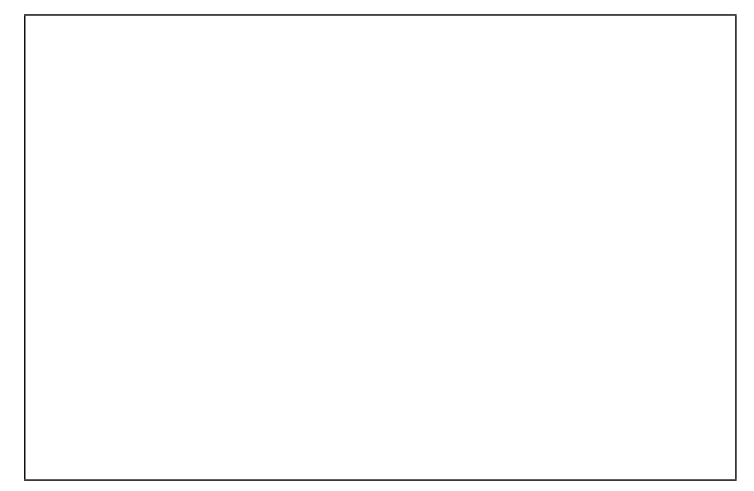

Fig. 1. The DSC signal from $\mathrm{LiTlSO}_{4}$ powder observed on a heating run. Two small anomalies are indicated by arrows. 
a large one at $288 \mathrm{~K}$. The amount of the enthalpy change $\Delta H$ was $0.017,0.027$ and $1.65 \mathrm{~kJ} / \mathrm{mol}$, respectively. The transition entropy at $288 \mathrm{~K}$ was $5.75 \mathrm{~J} /(\mathrm{mol} \mathrm{K}) \simeq R \ln 2.0$.

On the other hand, only one DSC signal was observed for the single crystal. It was found that the anomaly depended on the turning temperature at which a cooling run was changed to a heating run. If the crystal was cooled enough, say below $230 \mathrm{~K}$, then the single crystal sample showed a large anomaly at $288 \mathrm{~K}$ with $\Delta H=1.47 \mathrm{~kJ} / \mathrm{mol}$ on the heating. The excess heat flow amounts to the entropy change of $5.10 \mathrm{~J} /(\mathrm{mol} \mathrm{K}) \simeq R \ln 1.8$. Since the single crystal sample was very small, this value is not so accurate as the powder one.

On the contrary, if the turning temperature was above $250 \mathrm{~K}$, the small anomaly appeared at $267 \mathrm{~K}$ with $\Delta H \simeq 0.12 \mathrm{~kJ} / \mathrm{mol}$ and the anomaly at $288 \mathrm{~K}$ disappeared, as shown in Fig. 2 . If the

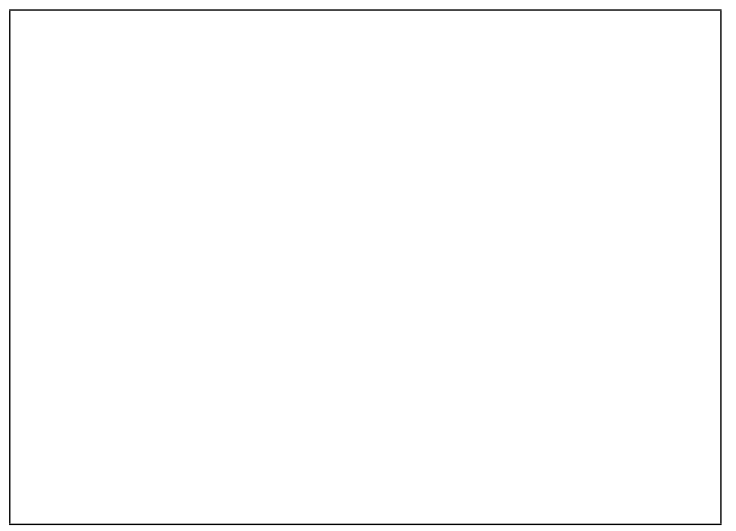

Fig. 2. The temperature dependence of the DSC signal from $\mathrm{LiTlSO}_{4}$ single crystal on heating runs. The sample temperature was changed from cooling to heating at $235 \mathrm{~K}$ (a), $245 \mathrm{~K}$ (b) and $260 \mathrm{~K}$ (c).

turning temperature was near the transition temperature of $243 \mathrm{~K}$, then weaker anomalies were detectable at $267 \mathrm{~K}$ and $288 \mathrm{~K}$. This result shows that once the low temperature phase is realized, it is easily super-heated and the intermediate phase does not appear on heating. If the crystal transforms to the room-temperature phase, then the intermediate phase appears on cooling. The phase sequence and the transition temperatures are summarized in Table I.

The transition temperatures and the shape of the anomaly depended slightly on the part of the crystal from which the sample was prepared. However, the transition at $267 \mathrm{~K}$, if it takes place, seems to be a second order one. Meanwhile the transition at $243 \mathrm{~K}$ (cooling) and $288 \mathrm{~K}$ (heating) is a first-order one with accompanying a large thermal hysteresis. The powder sample might be contaminated from a non-stoichiometric part synthesized by the Bridgman method. We attribute 
Table I. Phase sequence of $\mathrm{LiTlSO}_{4}$.

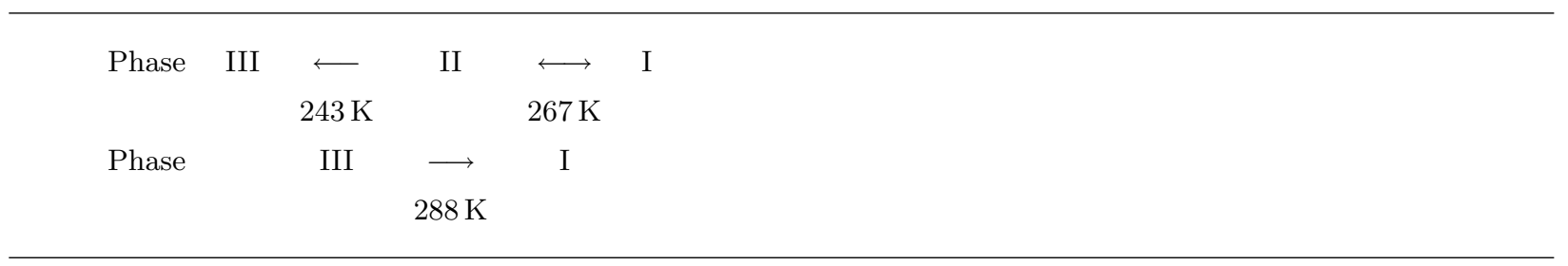

the additional small anomalies at $236 \mathrm{~K}$ and $263 \mathrm{~K}$ in the powder sample to such imperfection of the sample.

The transition entropy at $288 \mathrm{~K}$ on heating is almost $R \ln 2$ per one mole and the transition is an order-disorder type. Taking the $\mathrm{LiNH}_{4} \mathrm{SO}_{4}$ type structure into account, the $\mathrm{SO}_{4}$ tetrahedra are disordered in the room temperature phase of Pmcn. Each tetrahedron occupies, with an equal probability, two configurations related by the mirror symmetry perpendicular to the $a$ axis. In the low temperature phase, the tetrahedron occupies one configuration, dominantly. Then the expected entropy change is $R \ln 2$. If the lowest temperature phase is hexagonal, then the $\mathrm{SO}_{4}$ tetrahedra should have additional freedom to turn the apex upside down, which needs further entropy change of $R \ln 2$, and the total entropy change may be $R \ln 4$; however this is not the case.

Finally we note about the thermal anomaly at $267 \mathrm{~K}$, below which superstructure reflections were observed by the preliminary X-ray scattering study. ${ }^{11)}$ The estimated transition entropy is about $0.45 \mathrm{~J} /(\mathrm{mol} \mathrm{K})$, small enough to indicate a displacive type transition. On further cooling, the intensity of the supperstructure reflections increased discontinuously below $243 \mathrm{~K}$. Between $267 \mathrm{~K}$ and $243 \mathrm{~K}$, the cell dimension is fourfold along the $a$-axis. Below $243 \mathrm{~K}$, the unit cell volume becomes six times of the room-temperature phase. Therefore, the existence of the intermediate phase in $\mathrm{LiTlSO}_{4}$ is clear. A detailed diffraction study is now on progress.

\section{Acknowledgements}

The authors are grateful to Mr. Tsuchiyama for preparing the samples.

1) S. Sawada, M. Takashige, F. Shimizu, H. Suzuki and T. Yamaguchi: Ferroelectrics 169 (1995) 149 and references therein.

2) T. Mitsui, T. Oka, Y. Shiroishi, M. Takashige, K. Ito and S. Sawada: J. Phys. Soc. Jpn. 39 (1975) 845.

3) Y. Shiroishi, A. Nakata and S. Sawada: J. Phys. Soc. Jpn. 40 (1976) 911.

4) W. A. Dollase: Acta Crystallogr. B 25 (1969) 2298.

5) S. Tanisaki, H. Mashiyama, K. Hasebe, Y. Shiroishi and S. Sawada: Acta Crystallogr. B 36 (1980) 3084.

6) K. Itoh, H. Ishikura and E. Nakamura: Acta Crystallogr. B 37 (1981) 664. 
7) A. Kunishige and H. Mashiyama: J. Phys. Soc. Jpn. 56 (1987) 3189.

8) H. Mashiyama, K. Hasebe, S. Tanisaki, Y. Shiroishi and S. Sawada: J. Phys. Soc. Jpn. 47 (1979) 1198.

9) A. I. Kruglik, M. A. Simonov and K. S. Alexsandrov: Kristallografiya 23 (1978) 494.

10) F. Shimizu and M. Takashige: Ext. Abstr. (Autumn Meet., 1996) Physical Society of Japan, Part 2, p.75 [in Japanese].

11) S. Tsuchiyama: Master Thesis, Faculty of Scince, Yamaguchi University, Yamaguchi, 1997 [in Japanese].

12) A. Sawada, Y. Makita and Y. Takagi: J. Phys. Soc. Jpn. 41 (1976) 174. 


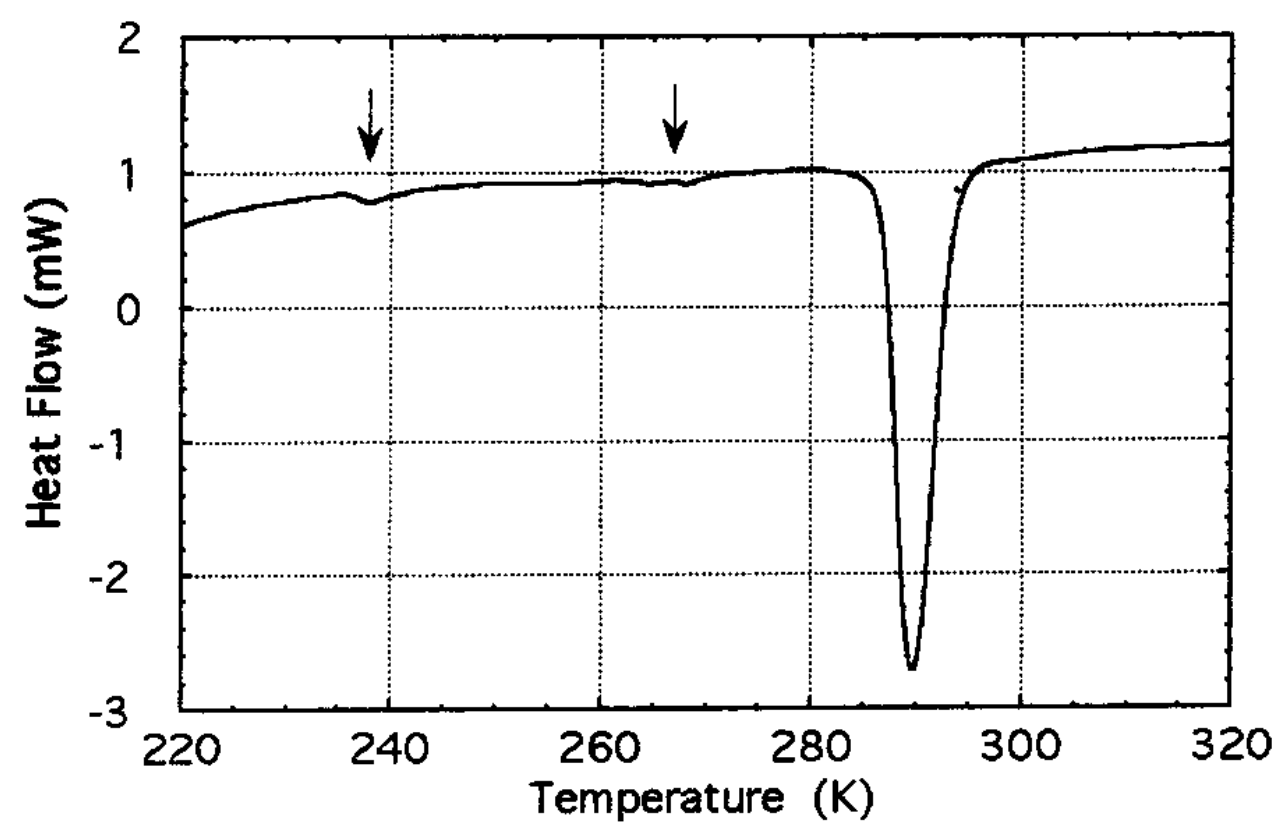

Fig. 1

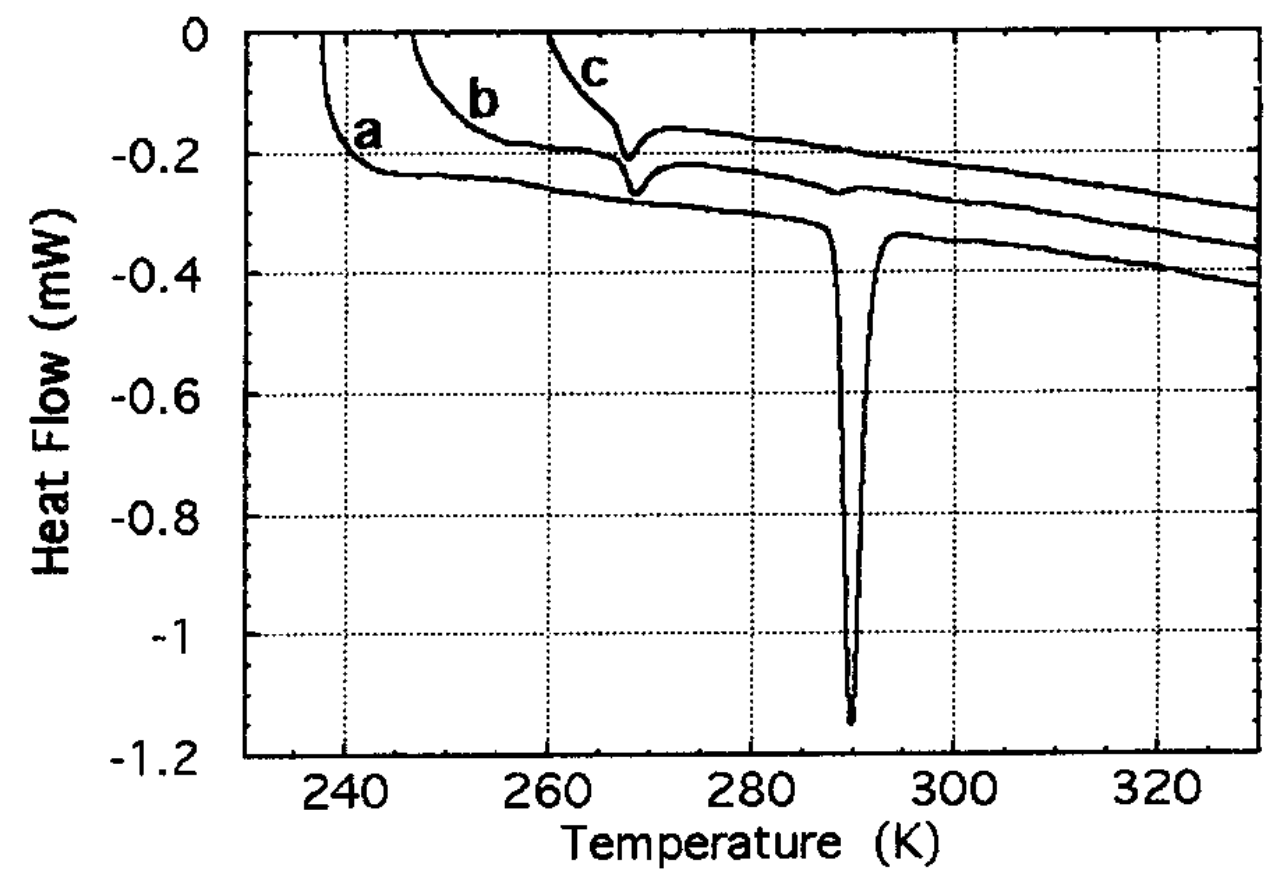

Fig. 2 